أثر الفساد على النمو الاقتصادي في مصر

The Impact of Corruption on Economic Growth in Egypt

\author{
محمد محمود فتح الله \\ معيد بقسم الاقتصاد \\ كلية التجارة - جامعة الزقازيق \\ fathala55@yahoo.com
}

د/ أشرف محمد السيد سيد أحمد

مدرس الاقتصاد

كلية التجارة - جامعة الزقازيق

economics125@yahoo.com 
تهدف الدراسة إلى قياس أثر الفساد على النمو الاقتصادي في الأجل الطويل في مصر خلال

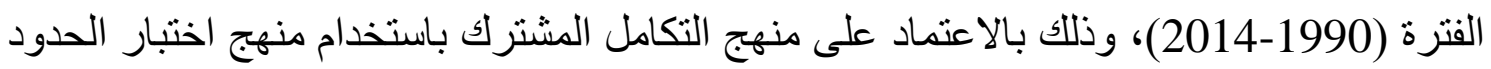
The Bounds Testing Approach الموزعة The Autoregressive Distributed Lag (ARDL)، وقد أظهرت النتائج وجود تأثثر إيجابي للفساد على النمو الاقتصادي باستخدام مؤشرين للفساد وهما مؤشري مدركات الفساد (CPI)، والتحكم في الفساد (CC) (Grease the Wheel)، مما يدعم فرضينما جاء تأثير مؤشر الفساد (ICRG) سلبي مما يدعم فرضية (Cand in the Wheels). و هذه النتيجة قد تم تأكيد ها بإجر اء اختبار Robust Least Squares (RLS) الكلمات الدالة: الفساد، النمو الاقتصادي، أسلوب ARDL، أسلوب RLS. 
يعتبر الفساد واحد من أقدم وأكثر الظواهر الموجودة في جميع الدول، فالتعريف الثائع للفساد

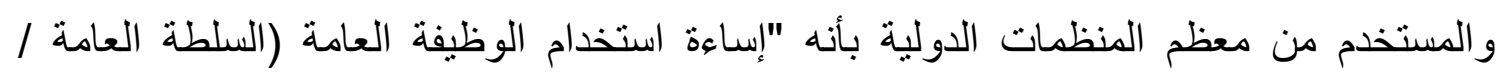

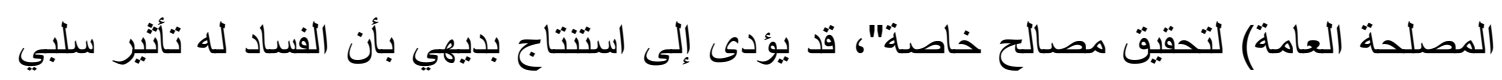

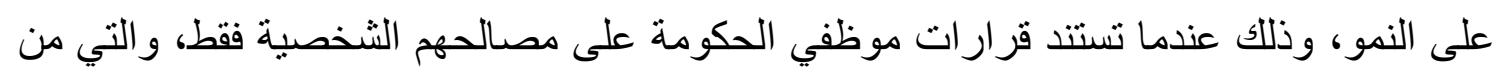

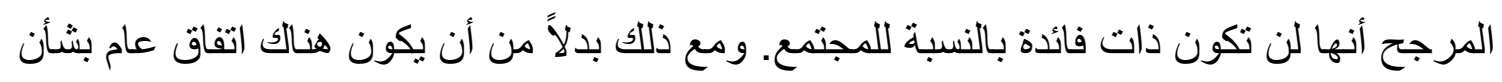

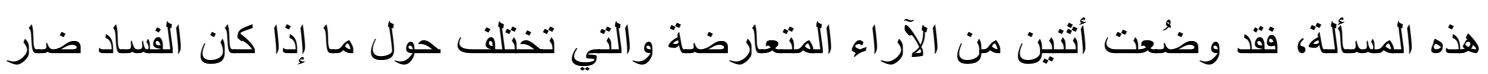

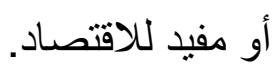

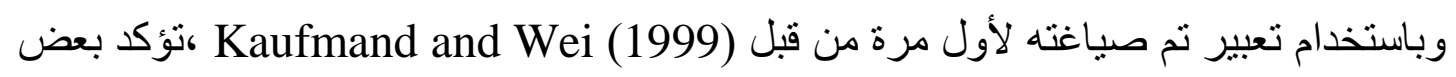

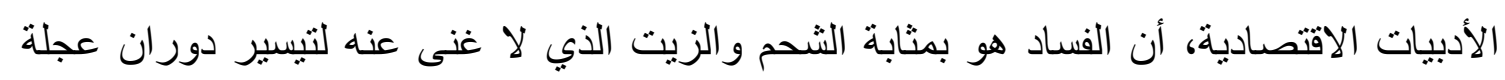

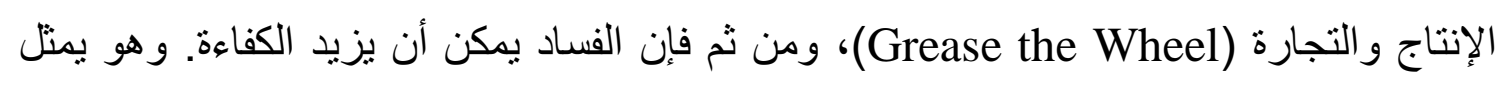

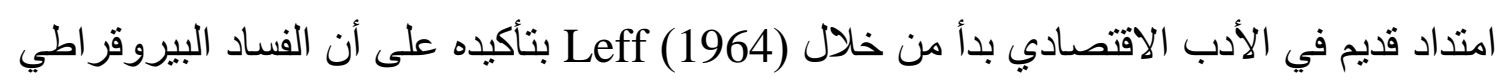

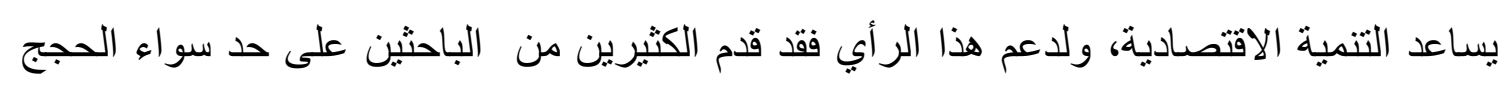

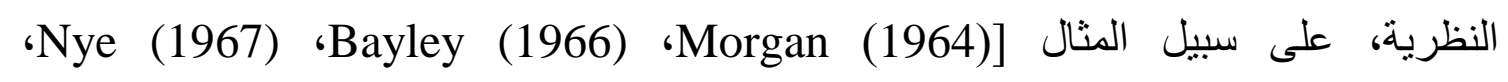
(2000) ‘Beck and Maher (1986) ،Lui (1985) ‘Huntington (1968)

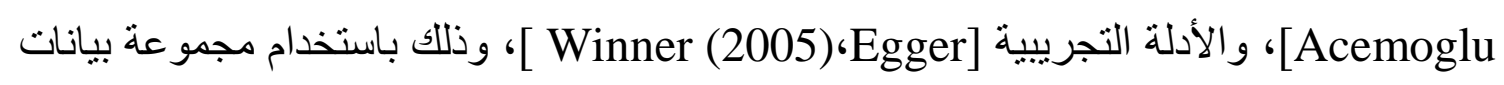
من 73 دولة من الدول النامية والأقل نمواً، حيث وجد أن الفساد يعمل على تحفيز الاستثمار

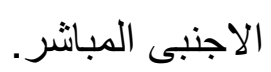

وقد استندوا في تبرير الثأثير الإيجابي للفساد، على قدرته على تجنب جمود الأنظمة الإدارية،

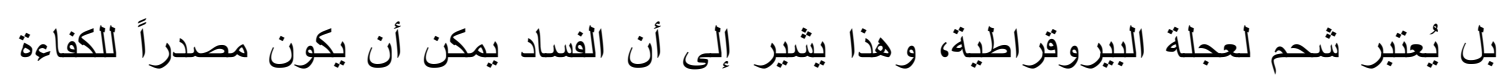

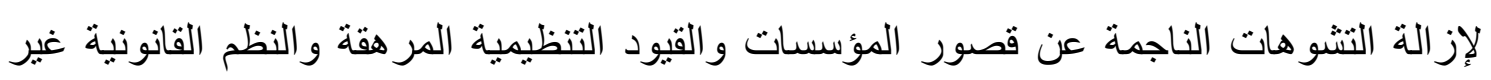

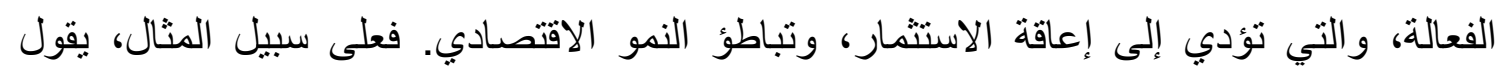

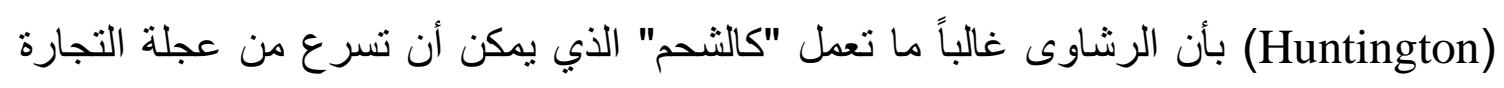

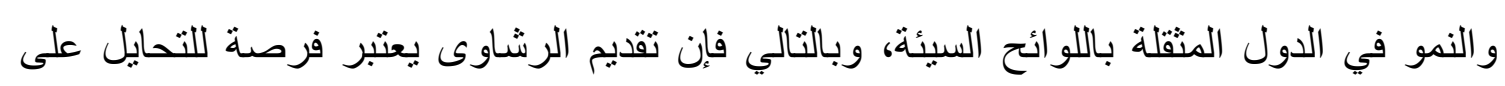
الرقابة وقواعد العمل الحكومية السيئة مما يجلب قدراً من تيسير الأعمال ورفع القيود الإدارية العاية 
و القانونية والفنية. كما يرى Morgen أن الفساد في الحالات القصوى لا يكون مر غوباً فيه فحسب بل ضروري للحفاظ على تنامي الاقتصاد. ولذلك فإن الفساد يعتبر "ثاني أفضل بديل" أو "كالثحم" و لاسيما في البيروقراطية العاجزة

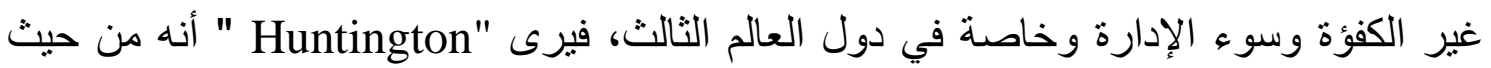

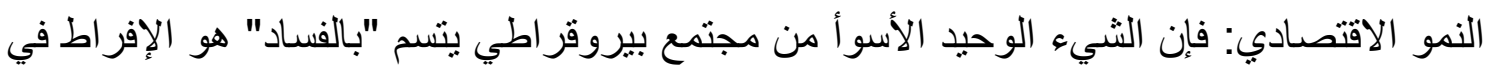
المركزية و البيروقر اطية الصادقة.

و على النقيض من Leff، نرى المدرسة المقابلة أن الفساد بمثابة رمال تعيق حركة عجلة النمو، من خلال فرضية (Sand in the Wheels)، وقد دعم هذه الفرضية الكثير من الباحثين مثل: [Adese- (1999) ; Ader) ; Murphy et al(1993) ; Tullock (1962)

.[Ackerman

فيرى Rose-Ackerman (1978) Kaufmann and Wei (1998) أن رأى Reff صحيح، عندما يَؤخذ سوء التنظيم ومرونة القو انين في الاعتبار ، حيث يُنتاح للبيروقر اطيين في كثير من الأحيان الكثير من الحرية، ليقرروا كم المضايقات التي سيفرضونها على الثركات من أجل

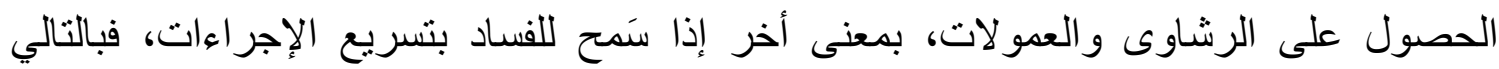

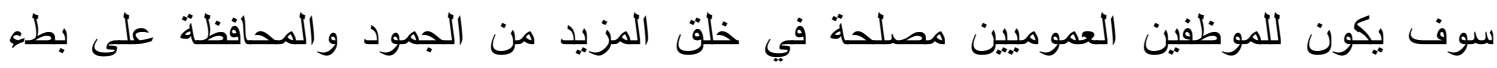
الإجراءات الإدارية للحصول على مزيد من الرشاوى. فعلى سبيل المثال، فإن مفتشي الضرائب بيعتمدوا على التقدير الجز افي للاخل الخاضع للضريبة، كما يمكن لمفتتي السلامة الصناعية تقرير

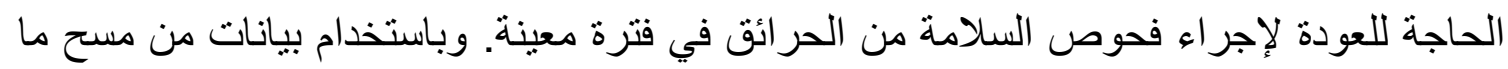

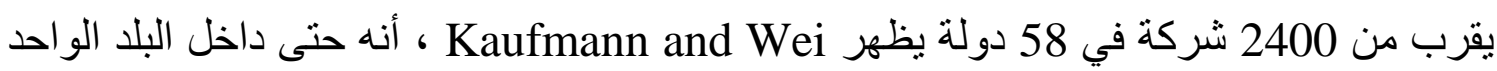

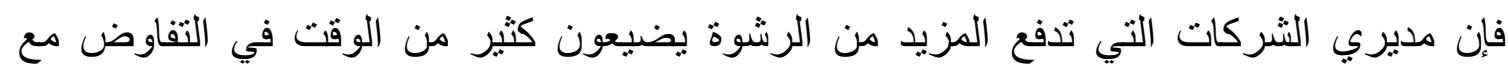

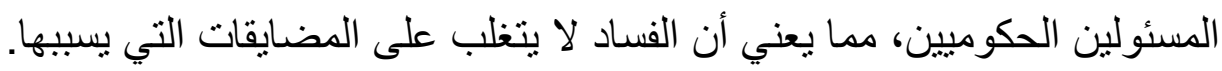
تتفثى ظاهرة الفساد في مصر - كحال الكثير من دول العالم - بمختلف صوره سواء كانت سياسية أو اقتصادية أو اجتماعية، فالحالة المصرية تكاد تكون نموذجية في دراسة كيفية تحول دول دهرل

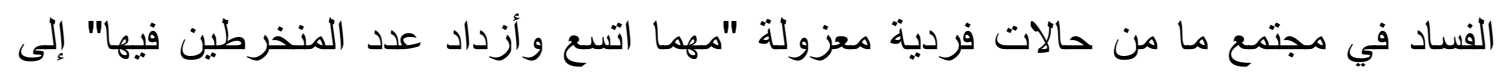
ممارسة مجتمعية شاملة بالمعنى الحقيقي لا المجازى للكلمة، حيث تعدت الممارسات التقليدية 
لتتمل مفاصل المجتمع الحساسة ومؤسساته وثقافته من خلال ظاهرتين يغذى كل منهما الأخر ،

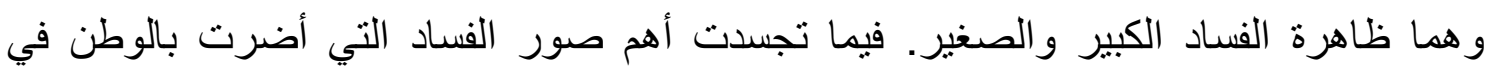

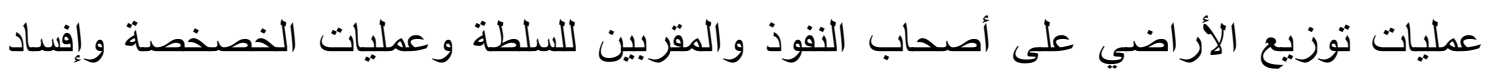
صناعة الغزل والنسيج وبيع ديون مصر وتصدير الثروات بأبخس الأثمان وانتشار الرشاوى ولثي و الوساطة و المحسوبية في مختلف المؤسسات الحكومية وانعدام العدالة في الأجور و غير ها الكثير. بيد أن هذا الواقع الجديد قد أتخذ طابعاً "مؤسسياً" منذ بداية التسعينات، فنو غلت جماعات

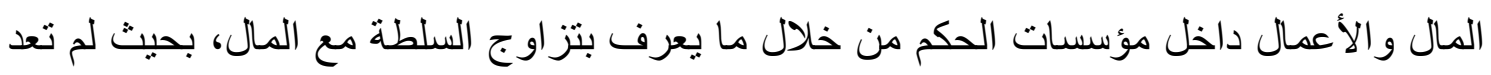

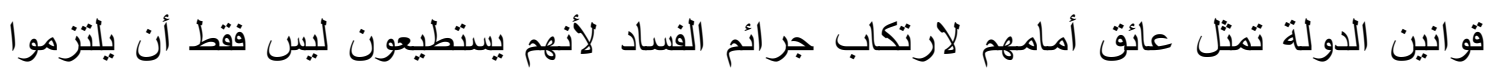

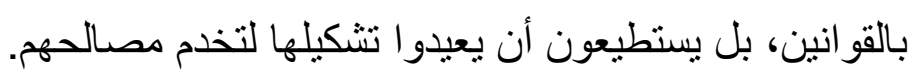
و هو ما أنعكس على وضع مصر داخل مؤشرات الفساد الدولية، فطبقاً لمؤشر مدركات الفساد

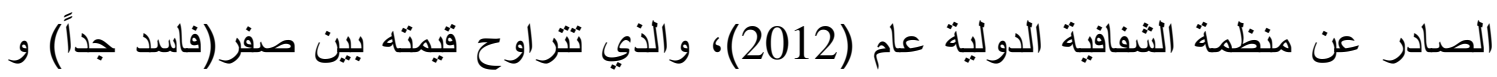
10(شفاف جداً) حصلت مصر على 3.2 درجة محتلة بذلك مرتبة متدنية جداً وهى 118 من 174 من 120

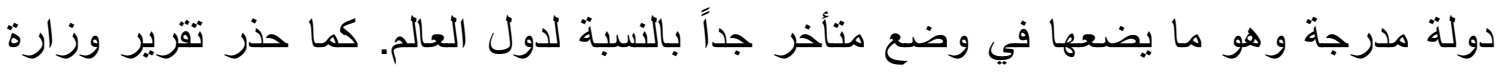
التجارة الأمريكية الثركات ورجال الأعمال الراغبين من الاستثمار في مصر من انتثار الفساد بها بشكل يمثل مخاطر جدية على استثمار اتهم ويفرض حالة من عدم اليقين.

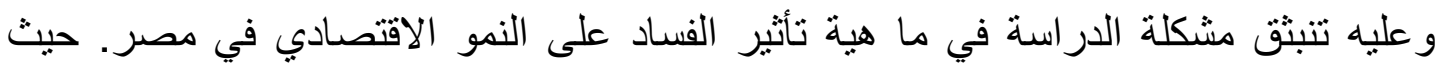

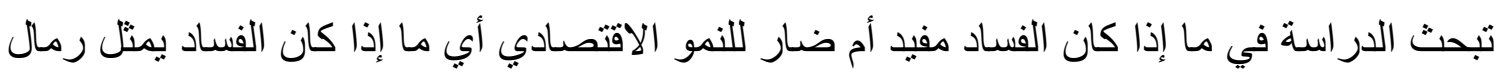
أم تثحيم لعجلة النمو الاقتصادي.

\section{(2) (2) (الدراسات السابقة:}

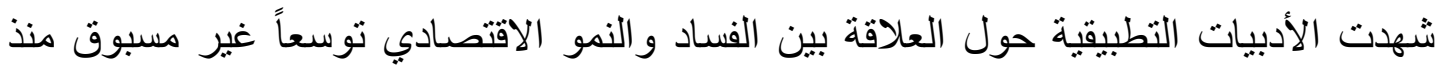

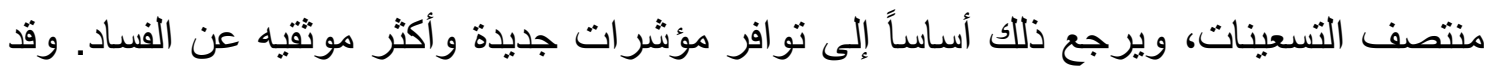
وجدت غالبية هذه الدراسات أن الفساد قد يكون له تأثير سلبي على النمو، ففي أول تحليل تطبيقي

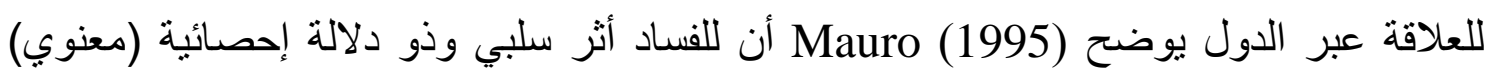
على معدل نمو نصيب الفرد من الناتج المحلى، وذلك لثريحة من 58 دولة خلال الفترة (1960) 
1985)، وقد وضح أن انخفاض الفساد بدرجة واحدة يؤدى لزيادة نصيب الفرد من الناتج بمقدار $\% 0.8$ وقد تأكدت هذه النتيجة في وقت لاحق من قبل العديد من الدراسات التطبيقية التي وجدت أدلة

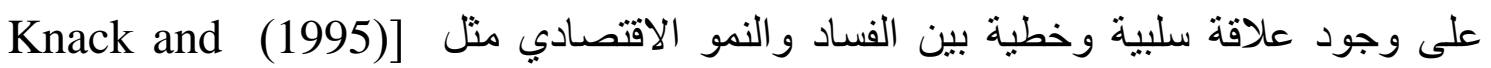
(2000) ‘Leite and Weidmann (1999) ،Tanzi (1998) ،Mauro (1997) ‘Keefer ‘Gyimah-Brempong (2006) Méon and Sekkat (2005) ،Tanzi and Davoodi Wisitsuwan ، et al. (2011)،Hodge ‘Dzhumashey (2009) ‘Bissessar (2008) ‘Dridi (2013) ‘Nuriyev and Hickson (2012) ،and Chintrakarn (2012)

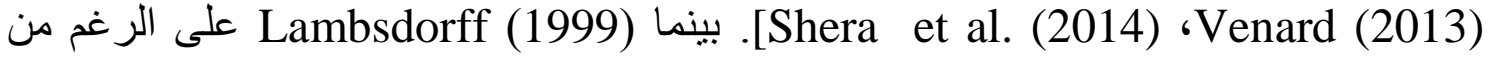
تأكيده على هذه العلاقة السلبية ولكنه برى أن استتناج العلاقة السبيية بينهما أمر صعب. فعلى سبيل المثال، يمكن أن يؤدى انخفاض النمو في بعض الدول النامية أو المتخلفة إلى جعل غالبية

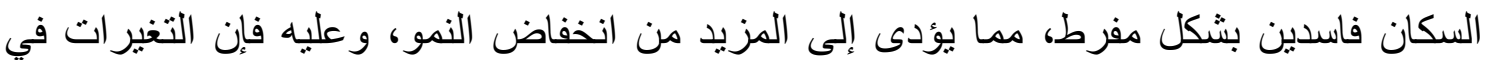
طبيعة الفساد وليس مستوى الفساد تعتبر من المحددات الهامة للنمو الاقتصادي. ومع ذلك فقد شكك بعض الباحثين في قوة العلاقة بين الفساد والنمو وخاصة، وإن الدراسات

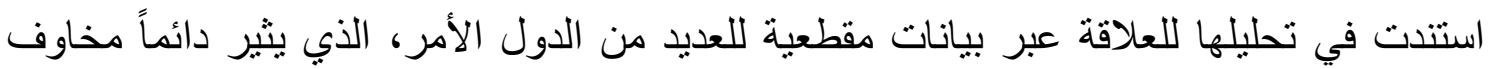
جدية حول عدم التجانس (unobserved heterogeneity) بين مشاهدات البيانات للادول المختلفة، فبعض الدراسات وجدت أن تأثثر الفساد على النمو ليس دائماً معنوي، بينما دراسات أخرى وجدت أن هذا التأثير حساس جداً لإضافة محددات أخرى للنمو. فعلى سبيل المثال، Kisunko and Weder (1998) ودراسة عزت (2011) و التي وجدت أن العلاقة بين الفساد و النمو لا تتسم بالاستقرار حيث تعتمد النتائج على طريقة التقدير ومؤشر الفساد الستخدم، فقد أنشارت النتائج إلى وجود علاقة موجبة بين الاثثين باستخدام طريقة المربعات الصغرى (OLS)، ولكن تحولت هذه العلاقة إلى سلبية بعد تغيير طريقة التقدير إلى (3SLS).

كذللك في العديد من الدراسات الأخرى نجد أن هذه العلاقة المعنوية تميل إلى الاختفاء عندما يتم إدراج متغيرات تكييفية (Conditioning Variables) أخرى في تحليل الانحدار، ويتضح ذلك بوضوح من خلال دراسة (2000) Abed and Davoodi، والتي هدفت إلى اختبار أهية 
الفساد ضد الإصلاحات الهيكلية في شرح أداء الاقتصاد الكلى بالنسبة للاقتصاديات التي تمر بمرحلة انتقالية. وبالتطبيق على 25 دولة خلال الفترة (1994-1998) وجدت الدراسة أن معامل الفساد يصبح معنوي عند إضافة مؤشر الإصلاحات الهيكلية إلى الانحدار، وقد تأكدت نفس النتيجة في دراسة Mauro (1995) ، حيث وجد أنه بعد السيطرة على الاستثمار أصبح تأثثير الفساد على النمو الاقتصادي غير معنوي. وقد قدم باحثين آخرين منهم (2001) Mo (2004) Pellegrini and Gerlagh من تأثير الفساد على النمو ينتقل بشكل غير مباشر من خلال المحددات الرئيسية للنمو والتي تتمثل أهمها في الاستثمار ور أس المال البشرى والاستقرار السياسي.

وفى سياق متصل، نجد أن الأدبيات التطبيقية الأكثر حداثة تبرز أن علاقة الفساد بالنمو لا يمكن تفسير ها دون الأخذ بعين الاعتبار الإطار المؤسسي للدولة. وقد وجدت عدد من الدراسات أن العلاقة بين الفساد والنمو الاقتصادي غير خطية، مما يثير إلى أن تأثير الفساد على النمو قد تختلف بين الدول وفقاً لنوعية الإعداد المؤسسي. فمثناً، يرى Braguinsky (1996) أن الفساد يلعب دور وظيفي في النمو الاقتصادي في الأسواق التنافسية، بينما في الأنظمة الثمولية يصبح الفساد أعمق وتمتد جذوره في النظام الاقتصادي والاجتماعي، ما يؤدى في النهاية إلى انهيار هذا النظام ويجعل من الصعب على هذه الدول محاولة الحفاظ على أو تحسين معدل النمو الاقتصادي.

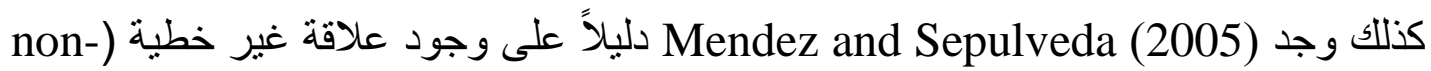
(monotonic بين الفساد والنمو، وذكر أن الفساد له تأثير مفيد في الدول المتمتعة بدرجات عالية من الحرية السياسية، بينما في أماكن أخرى نجد تأثير الفساد على النمو ليست قوية. وقد قام بدر اسة العلاقة عن طريق تقسيم عينة الدول إلى مجمو عتين حسب درجة الحكم الرشيد لديها، ووجدت أنه في الدول التي تمتلك مؤسسات عالية الجودة والكفاءة، يكون للفساد فيها تأثير سلبي كبير على النمو، بينما في البلدان ذات المؤسسات الضعيفة والأقل جودة، لا نلاحظ أي تأثير للفساد فيها على النمو الاقتصادي. وقد تم بحث الدور الحاسم للمؤسسات في تحديد علاقة الفساد بالنمو في الآونة الأخيرة من قبل حيث قدما دليل على أن الفساد يكون أقل ضرراً بكثير في الدول التي تمتلك إطار مؤسسي أقل فعالية. ويؤكد هذه التتيجة التي تبدو أنه في صالح منظور كفاءة 
الفساد في مساعدته لتجاوز أوجه القصور المؤسسية القائمة. أيضاً أظهرت دراسة (2010) Heckelman and Powell

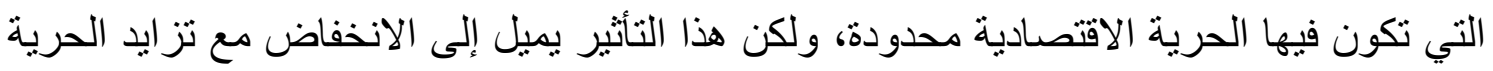
الاقتصادية.

وفى محور أخر حاولت العديد من الدراسات الإجابة على تساؤل لماذا النمو الاقتصادي لبعض

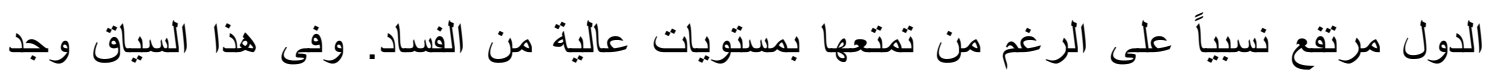

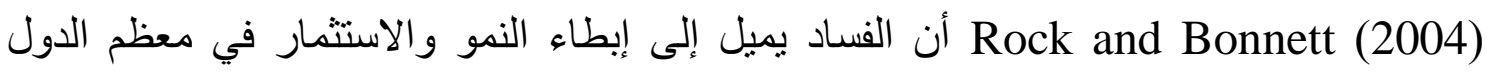

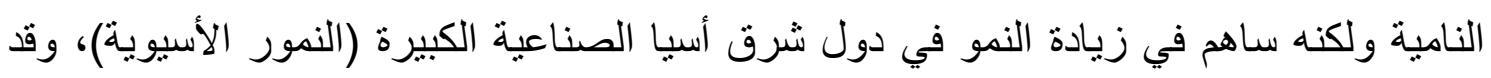
أكد النتيجة (2008) Ali and Comm باستخدام بيانات 33 دولة خلال الفترة (1986-1996)،

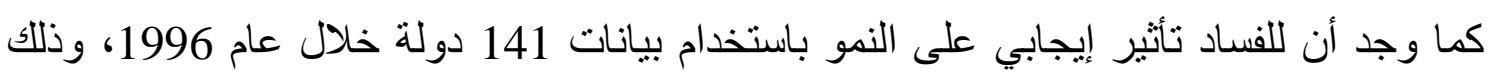

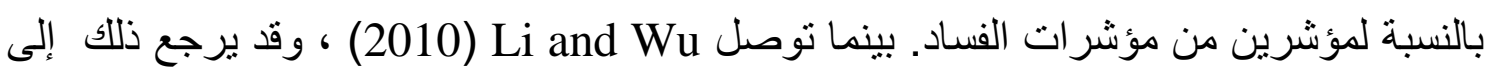
ارتباط التأثثر السلبي للفساد بالمستوى العام للتقة في المجتمع، فوجود مستوى أعلى من الثقة يقلل من التأثير السلبي للفساد على التنمية الاقتصادية. ونستخلص من ذللك، اختلاف الدراسات التطبيقية حول طبيعة العلاقة بين الفساد والنمو الاقتصادي. فعلى الرغم من أن العديد من الدراسات وجدت تأثثر سلبي للفساد مُدعمة بذلك فئكة فرضية (Sand in the Wheel)، إلا أن قلة من الدراسات قد وجدت نأثير إيجابي للفساد على الثى

النمو وخاصة في دول شرق أسيا مما يدعم فرضية (Grease in the Wheel).

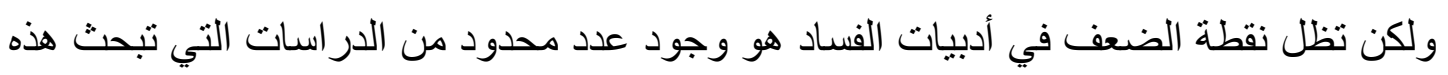

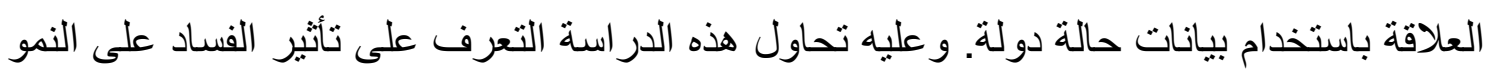

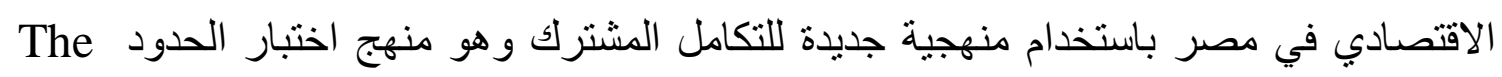
Bounds Testing Approach الموزعة The Autoregressive Distributed Lag (ARDL). وباستخدام (RLS) . Robust Least Squares (3) توصيف البيانات: تعتمد الدراسة على بيانات سلاسل زمنية سنوية لدصر خلال الفترة من 1990 إلى 2014، وقد تم التعبير عن النمو الاقتصادي باستخدام مؤشر متوسط نصيب الفرد من الناتج المحلى لنى 
الإجمالي بالأسعار الثابتة للدولار الأمريكي لعام 2005 (GDPc) الصادرة عن قاعدة بيانات البنك الدولى. ويتم التعبير عن مستوى الفساد بثلاثة مؤشرات، وذللك للتحقق من قوة النتائج

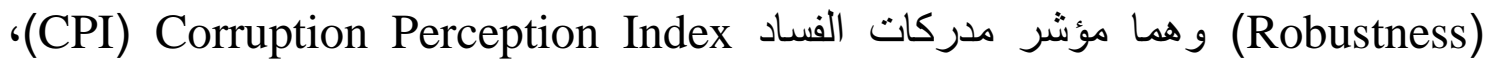
ومؤشر التحكم في الفساد Control of Corruption (CC)، ومؤشر الفساد من الدليل الدولى مونى للمخاطر القطرية ICRG) International Country Risk Guide). فبالنسبة لمؤشر مدركات الفساد (CPI)، فتقوم بإصداره منظمة الثفافية الدولية منذ عام

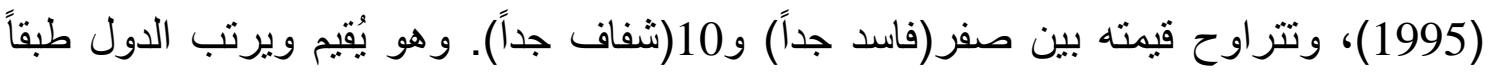
لارجة إدر الك الفساد في القطاع العام، وهو مؤشر مركب ويشكل مزيجاً من استطلاعات الر أي. ويعتمد على بيانات ذات صلة بالفساد، نم جمعها عن طريق استقصاءات متخصصة، قامت بها

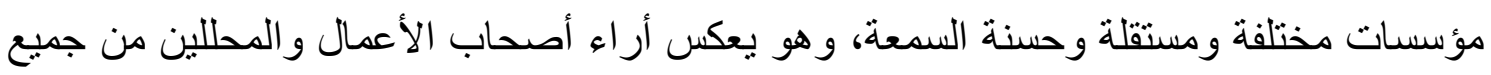
أنحاء العالم، متضمناً المتخصصين و الخبراء من نفس الدولة الجاري تقييمها. أما مؤشر التحكم في الفساد (CC)، فيصدره البنك الدولى كأحد المؤشرات الفرعية "للمؤشرات

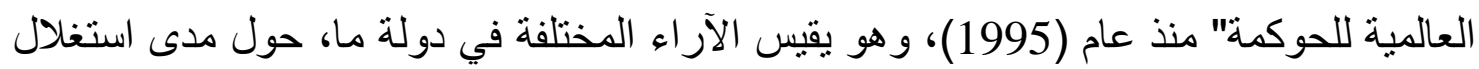
السلطة العامة في تحقيق مكاسب شخصية، وثقة المواطنين في الموظفين الحكوميين و السياسيين، بالإضافة إلى مدى انتشار أثنكال الفساد الصغير والكبير خاصة في الأحزاب السياسية، و الإعلام، والجهاز الإداري، والبرلمان، والقضاء، وأخيراً مدى توفر الثفافية وآليات الدساءلة و المحاسبة. ويستند المؤشر على 22 مصدر أساسي للبيانات لمعرفة التصورات عن الفساد من عدد كبير من المشاركين في الاستطلاع وتقييمات الخبراء في جميع أنحاء العالم و المنظمات غير لهير

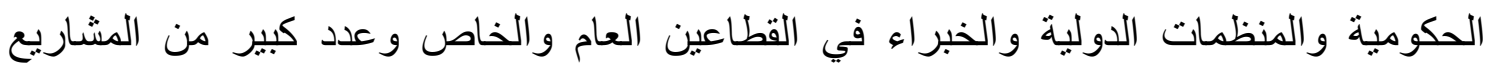
و المسوح. وتقدير الفساد فيه ينراوح بين صفر (فاسد جداً) و 100 (نظيف جداً). وأخيراً مؤشر الفساد (ICRG)، فيتم إصداره بواسطة مجموعة وحدة خدمات المخاطر منذ عام (PRS) Political Risk Services Inc.

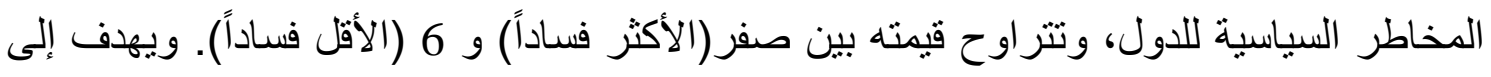
تقييم درجة الفساد السائدة في كل بلد استنادا على دراسات استقصائية بين المستثمرين الأجانب.

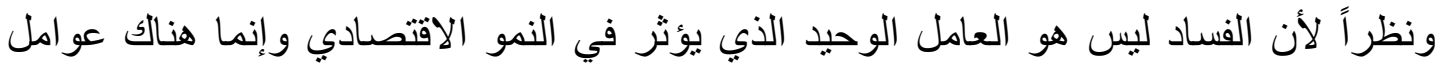
أخرى، فقد تم استخدام مجموعة من المتغيرات المساعدة أو الحاكمة (Control Variables) 
و التي تم اختيارها بما ينسجم مع الأدبيات السابقة، والتي وضحت أن التأثير غير المباثر للفساد على النمو يتم من خلال تأثير الفساد على محددات الاديات النمو ومن أهمها الاستثمار ورأس المال

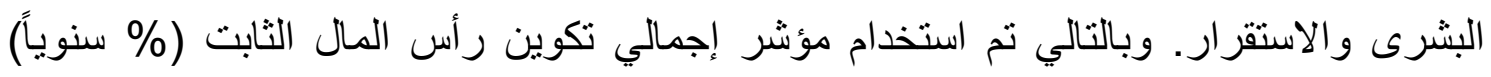
(GFCF) الخارجية والصادران من البنك الدولى، ومؤشر التنمية البشرية (HDI) من برنامج الأمم المتحدة

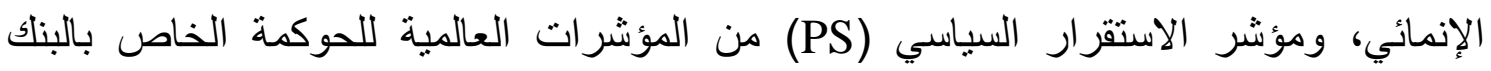

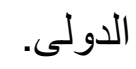
كذلك نظراً لان مؤشرات (CPI)، (CC))، (PS) لم تكن متاحاً قبل عام 1995 فقد نم الاعتماد

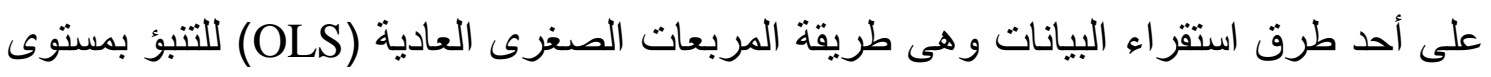

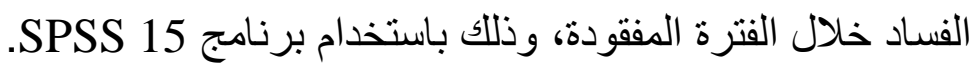
ويوضح الجدولين (1)، (2) توصيف إحصائي عام للمتغيرات المستخدمة ومصفوفة الارتباط بين المتغيرات الرئيسية على الترتيب. جدول (1): توصيف عام لمتغيرات الدراسة

\begin{tabular}{|c|c|c|c|c|c|}
\hline & $\mathbf{N}$ & Mean & Std. Dev & Max & Min \\
\hline GDPc & 25 & 1207.6 & 249.03 & 1575.93 & 872.37 \\
\hline CPI & 25 & 3.16 & 0.229 & 3.7 & 2.8 \\
\hline $\mathrm{CC}$ & 25 & 42.54 & 10.21 & 57.77 & 27.18 \\
\hline ICRG & 25 & 2.18 & 0.73 & 4.0 & 1.5 \\
\hline GFCF & 25 & 3.89 & 10.22 & 23.77 & -13.36 \\
\hline HDI & 25 & 0.598 & 0.059 & 0.694 & 0.502 \\
\hline TRD & 25 & 30.43 & 7.99 & 45.82 & 18.64 \\
\hline PS & 25 & 30.62 & 14.27 & 52.01 & 6.60 \\
\hline
\end{tabular}

جدول (2): مصفوفة الإرتباط بين المتغيرات الرئيسية للاراسة

\begin{tabular}{ccccc}
\hline \hline & GDP c & CPI & CC & ICRG \\
\hline \hline GDPc & 1 & & & \\
CPI & -0.023 & 1 & & \\
CC & -0.901 & 0.026 & 1 & \\
ICRG & -0.469 & -0.144 & 0.466 & 1 \\
\hline \hline
\end{tabular}




\section{(4) نموذج الاراسة:}

تعتمد الدراسة في قياس وتقدير أثر الفساد على النمو الاقتصادي في مصر على نموذجين وذلك

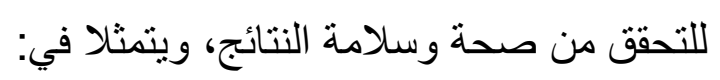

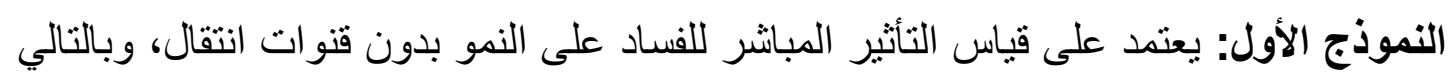

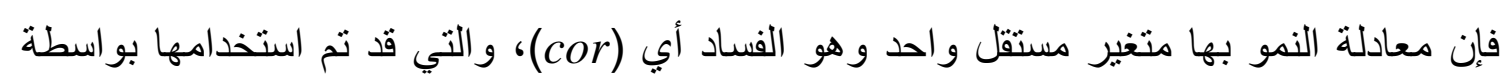

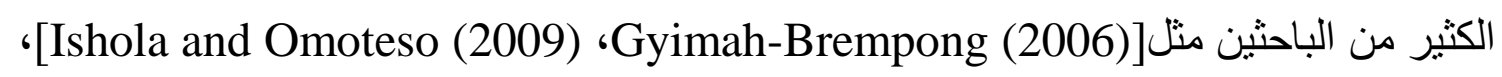

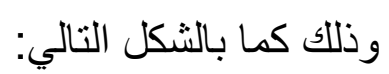

$$
g d p c_{t}=\beta_{0}+\beta_{1} \operatorname{cor}_{t}+\varepsilon_{\mathrm{t}}
$$

أما النموذج الثاني: فهو يعتمد في قياس تأثير الفساد على النمو من خلال قنوات انتقال وذللك

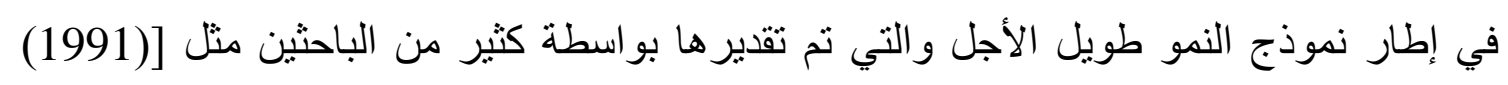
Gyimah- (2006) ،Méndez \& Sepúlveda (2006) ،Caselli et al. (1996) ،Barro [Brempong] ، ويمكن توضيح الخطوط العريضة لمعادلتها من خلال أدبيات اقتصاديات التتمية، والتي تثبر إلى أن الفساد يقلل نمو الدخل من خلال تخفيض الاستثمار في كل من رأس المال

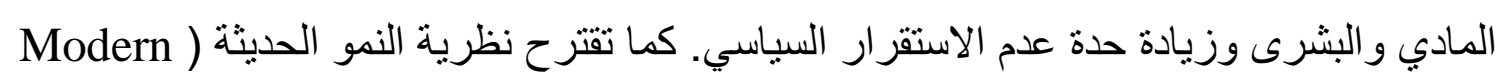
(Growth Theory

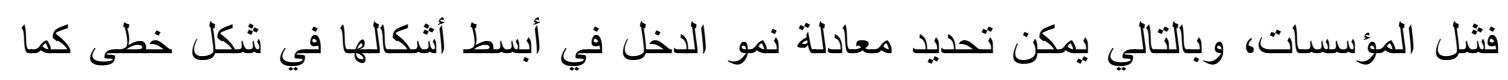

$g d p c_{t}=\beta_{0}+\beta_{1} \operatorname{cor}_{t}+\beta_{2} g f c f_{t}+\beta_{3} h d i_{t}+\beta_{4} t r d_{t}+\beta_{5} p s_{t}+\varepsilon_{\mathrm{t}}$ (2) حيث نفترض أن نصيب الفرد من الناتج (gdpc) يعتمد على معدل نمو إجمالي تكوين رأس

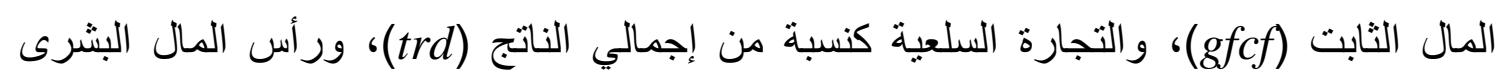

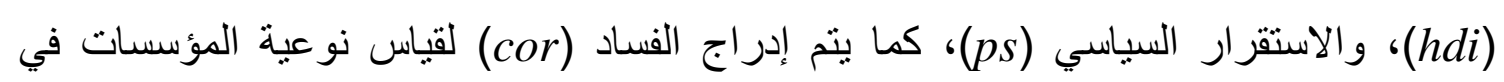


تعتمد الدراسة في تحليل السلاسل الزمنية وقياس أثر الفساد على النمو الاقتصادي في مصر، وذلك باستخدام برنامج التحليل الإحصائي 9.0 E-Vews على الخطوات التالية:

(1-5) فحص سكون السلاسل الزمنية باستخدام اختبار (ADF)

لاستخدام نماذج انحدار السلاسل الزمنية المختلفة، ينبغي أن تكون هذه السلاسل ساكنة، وذلك من أجل تجنب الانحدار الزائف، وبالتالي فالخطوة الأولى في التحليل هو التحقق من سكون هذه السلاسل وتحديد درجة تكامل كل سلسلة في النموذج للتأكد من أنها ليست ساكنة في الفروق الثانية

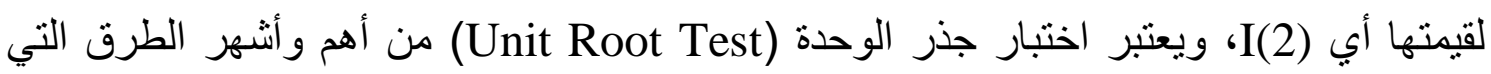

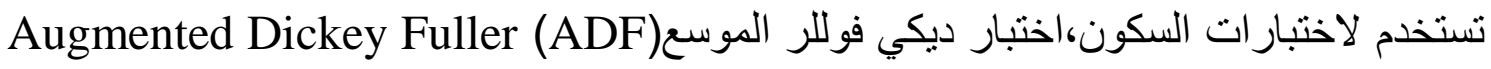
ويعد الأكثر استخداما في البحوث التطبيقية للكثف عن السكون، ويلخص الجدول (3) نتائج اختبار جذر الوحدة باستخدام اختبار (ADF).

جدول (3): نتائج اختبار Unit root test باستخدام ADF

\begin{tabular}{|c|c|c|c|c|c|}
\hline \multirow[b]{2}{*}{$\begin{array}{c}\text { Variable } \\
\mathrm{s}\end{array}$} & \multicolumn{2}{|c|}{ Level } & \multicolumn{2}{|c|}{ First difference } & \multirow{2}{*}{ 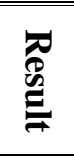 } \\
\hline & Intercept & $\begin{array}{c}\text { Intercept \& } \\
\text { trend }\end{array}$ & Intercept & $\begin{array}{l}\text { Intercept } \\
\text { \& trend }\end{array}$ & \\
\hline GDPc & $-1.07(0.709)$ & $-3.73(0.042)^{* *}$ & & & $\mathbf{I}(\mathbf{0})$ \\
\hline CPI & $-4.51(0.003)^{*}$ & & & & $\mathbf{I}(\mathbf{0})$ \\
\hline $\mathrm{CC}$ & $-1.45(0.539)$ & $-3.15(0.118)$ & $-6.11(0.000)^{*}$ & & $\mathbf{I}(\mathbf{1})$ \\
\hline ICRG & $-1.61(0.464)$ & $-2.12(0.512)$ & $-3.44(0.019)^{* * *}$ & & $\mathbf{I}(\mathbf{1})$ \\
\hline GFCF & $-3.47(0.018)^{* *}$ & & & & $\mathbf{I}(\mathbf{0})$ \\
\hline HDI & $-0.21(0.925)$ & $-2.17(0.482)$ & $-5.06(0.000)^{*}$ & & $\mathbf{I}(\mathbf{1})$ \\
\hline TRD & $-1.29(0.619)$ & $-2.17(0.479)$ & $-3.45(0.019) * *$ & & $\mathbf{I}(\mathbf{1})$ \\
\hline PS & $-0.92(0.763)$ & $-2.69(0.247)$ & $-4.28(0.004)^{*}$ & & $\mathbf{I}(\mathbf{1})$ \\
\hline $\begin{array}{c}\text { Critical } \\
\text { Values }\end{array}$ & & & First diff & ence & \\
\hline$\% 1$ & -3.7379 & -4.3098 & -3.6999 & -4.4163 & \\
\hline$\% 5$ & -2.9919 & -3.5742 & -2.9763 & -3.6220 & \\
\hline$\% 10$ & -2.6355 & -3.2217 & -2.6274 & -3.2486 & \\
\hline
\end{tabular}


ويتضح من نتائج الجدول أن السلاسل الزمنية المستخدمة تختلف في رتبة سكونها، حيث نجد

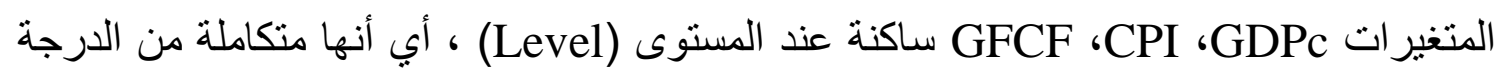
أمند مستوى معنوية 5(0)

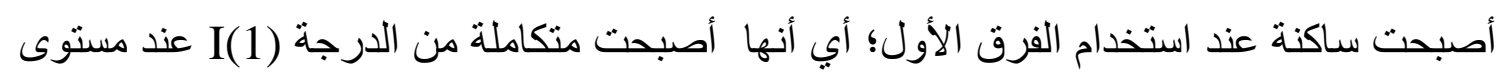

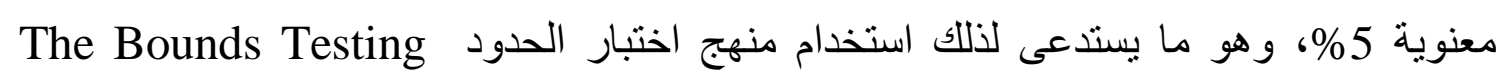

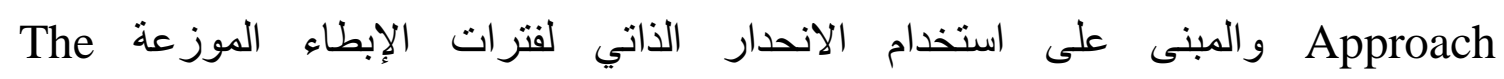
Autoregressive Distributed Lag (ARDL)

:ARDL (ختبار التكامل المشترك (Co-integration) باستخذام منهج) (2-5) لإجر اء التكامل المشترك بين المتغيرات طبقاً لمنهج ARDL ، ينت فيه أولاً اختبار ما إذا كانت

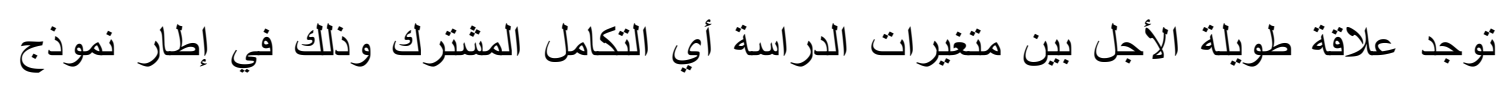

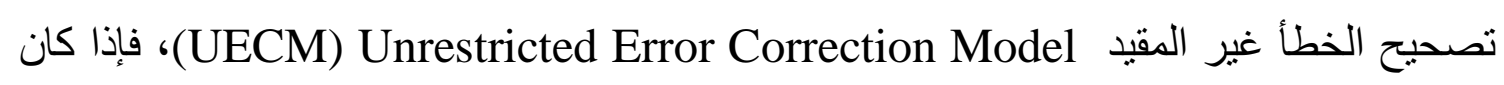
هناك تكامل مشترك فإننا نستطيع إكمال التحليل لتقدير معلمات الأجل الطويل والقصير.

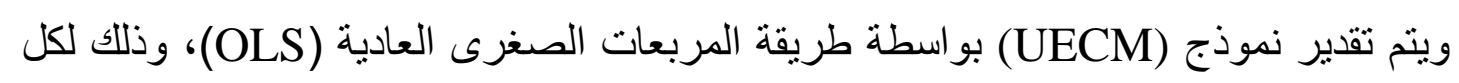
معادلة كالآتي: $\Delta \ln Y_{t}=\propto_{0 Y_{t}}+\sum_{i=1}^{m} \beta_{i} \Delta \ln Y_{t-i}+\sum_{i=0}^{n} \lambda_{i Y_{t}} \Delta \ln X_{t-i}+\varphi Y_{t-1}+\delta X_{t-1}+u_{t}(2)$ حيث يمثل Y المتغير التابع و متجه المتغير ات المستقلة، ويشير كل من ג، م إلى معاملات الأجل القصير (تصحيح الخطأ) بينما ب،

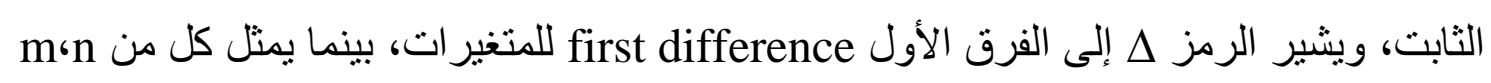

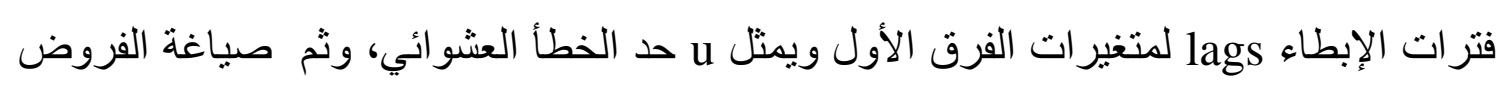

$$
\begin{aligned}
& H_{0}: \varphi_{1}=\delta_{2}=0 \quad \text { الفرض العدمى: عدم وجود تكامل مشترك بين المتغيرات } \\
& \text { الفرض البديل: وجود تكامل مشترك بين المتغيرات }
\end{aligned}
$$

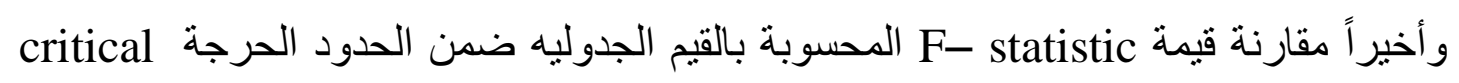
bounds 
معياري، فإن هناك قيمتين حرجتين لإحصاء هذا الاختبار: قيمة الحد الأدنى Lower Critical Bounds (LCB)

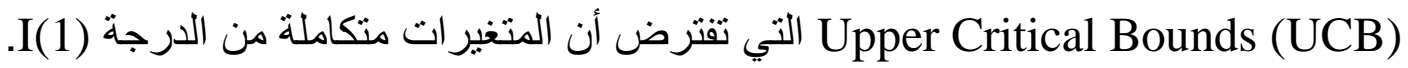
فإذا كانت قيمة F- statistic المحسوبة أكبر من قيمة الحد الأعلى الجدوليه (F > Fل)، فقي هذه الحالة يتم رفض الفرض العدمى وقبول الفرض البديل؛ أي أن هناك علاقة تكامل مشترك بين المتغيرات. وعلى النقيض من ذللك، إذا كانت قيمة F- statistic المحسوبة أقل من قيمة الحد الأدنى الجدوليه، ففي هذه الحالة يتم قبول الفرض العدمى الذي يشير إلى عدم وجود نكامل

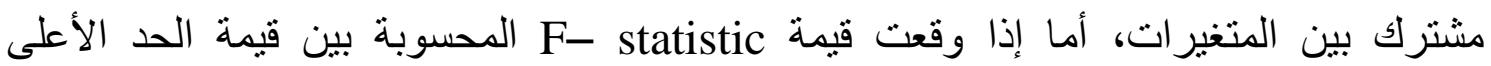
والأدنى، ففي هذه الحالة تكون النتيجة غير محسومة بمعنى عدم القدرة على اتخاذ قرار لتحديد عما إذا كان هنالك تكامل مشترك بين المتغيرات من عدمه، ويوضح الجدول رقم (4) نتائج اختبار

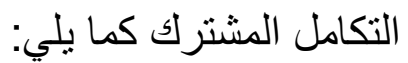

جدول (4): نتائج اختبار التكامل المشترك باستخدام نموذج (ARDL)

\begin{tabular}{|c|c|c|c|c|c|c|c|}
\hline \multicolumn{7}{|c|}{ Regressors: } & \multirow{2}{*}{\begin{tabular}{|c}
$\begin{array}{c}\text { F - } \\
\text { statistic }\end{array}$ \\
$\mathbf{5 . 0 8 9} * *$ \\
\end{tabular}} \\
\hline \multirow{3}{*}{ 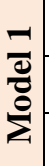 } & 1 & \multicolumn{5}{|c|}{ 1) $،$ ARDL $(2 \cdot G D P c=f(C P I)$} & \\
\hline & 2 & \multicolumn{5}{|c|}{ 2) ‘ ARDL (2،GDPc $=f(C C)$} & $12.412 *$ \\
\hline & 3 & \multicolumn{5}{|c|}{ 0)‘ ARDL (3،GDPc $=f(I C R G)$} & $5.910 * *$ \\
\hline \multirow{3}{*}{$\frac{N}{\grave{d}}$} & 4 & \multicolumn{5}{|c|}{ 1)‘ 1، 0، 0، 1، ARDL (1، PS)، TRD، HDI، GFCF،GDPc $=f(C P I$} & 8.098* \\
\hline & 5 & \multicolumn{5}{|c|}{ 2)، 0،2،2، 2، ARDL (1، PS)، TRD، HDI، GFCF،GDPc $=f(C C$} & $64.073 *$ \\
\hline & 6 & \multicolumn{5}{|c|}{$\begin{array}{l}\text { ، 2، 2، 2، 2، ARDL (1، PS)، TRD، HDI، GFCF،LGDPc = f(ICRG } \\
\text { 1) }\end{array}$} & $54.523 *$ \\
\hline \multirow{3}{*}{\multicolumn{2}{|c|}{ 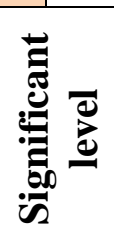 }} & \multicolumn{6}{|c|}{ Critical values bounds } \\
\hline & & \multicolumn{3}{|c|}{ Lower Critical Bounds (LCB) } & \multicolumn{3}{|c|}{ Upper Critical Bounds (UCB) } \\
\hline & & $\begin{array}{c}\text { Intercep } \\
\mathbf{t} \\
\text { \&Trend }\end{array}$ & $\begin{array}{c}\text { Intercept } \\
\text { \&no } \\
\text { Trend }\end{array}$ & $\begin{array}{c}\text { Trend \&no } \\
\text { Intercept }\end{array}$ & $\begin{array}{c}\text { Intercep } \\
\mathbf{t} \\
\text { \&Trend }\end{array}$ & $\begin{array}{c}\text { Intercept } \\
\text { no \& } \\
\text { Trend }\end{array}$ & $\begin{array}{c}\text { Trend \&no } \\
\text { Intercept }\end{array}$ \\
\hline \multirow{2}{*}{\multicolumn{2}{|c|}{$\begin{array}{c}\% 1 \\
\% 2.5\end{array}$}} & 6.1 & 4.94 & 3.69 & 6.73 & 5.58 & 5.39 \\
\hline & & 5.3 & 4.18 & 3.53 & 5.83 & 4.79 & 5.01 \\
\hline \multirow{2}{*}{\multicolumn{2}{|c|}{$\% 5$}} & 4.68 & 3.62 & 3.13 & 5.14 & 4.16 & 4.62 \\
\hline & & 4.05 & 3.02 & 2.58 & 4.49 & 3.02 & 3.84 \\
\hline
\end{tabular}

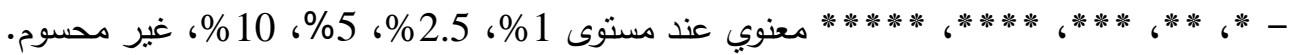

ويتبين من النتائج الموضحة أعلاه أن قيمة إحصاء (F- statistic) المحسوبة لكل النماذج تفوق قيمة الحد الأعلى الجدوليه (UCB) المناظرة ومن ثم يتم رفض فرض العدم وقبول الفرض الفه 
البديل بما يفيد وجود علاقة توازنية طويلة الأجل بين المتغيرات في هذه المعادلات أي تكامل مشترك. وبناء على ذلك يمكننا تقدير العلاقة طويلة الأجل وقصيرة الأجل بين الفساد والنمو كما يتضح من الجدولين (5)، (6) على الترتيب، كما يلي:

جدول (5): نتائج تقدير معاملات الأجل الطويل حسب معيار SBC

\begin{tabular}{|c|c|c|c|c|c|c|}
\hline & \multicolumn{3}{|c|}{ Model (1) } & \multicolumn{3}{|c|}{ Model (2) } \\
\hline & CPI & $\mathrm{CC}$ & ICRG & CPI & $\mathrm{CC}$ & ICRG \\
\hline Corruption & $\begin{array}{l}-158.81 \\
(0.005)^{*}\end{array}$ & $\begin{array}{c}-25.13 \\
(0.000)^{*}\end{array}$ & $\begin{array}{c}26.85 \\
(0.003)^{*}\end{array}$ & $\begin{array}{c}-187.50 \\
(0.015)^{* *}\end{array}$ & $\begin{array}{c}-15.91 \\
(0.015)^{* *}\end{array}$ & $\begin{array}{c}71.95 \\
(0.012)^{* *}\end{array}$ \\
\hline GFCF & & & & $\begin{array}{c}2.64 \\
(0.085)^{* * * *}\end{array}$ & $\begin{array}{c}21.08 \\
(0.020)^{* * *}\end{array}$ & $\begin{array}{c}8.61 \\
(0.007)^{*}\end{array}$ \\
\hline HDI & & & & $\begin{array}{l}2425.16 \\
(0.292)\end{array}$ & $\begin{array}{l}1823.83 \\
(0.000)^{*}\end{array}$ & $\begin{array}{l}4730.53 \\
(0.000)^{*}\end{array}$ \\
\hline TRD & & & & $\begin{array}{c}2.67 \\
(0.048)^{* * *}\end{array}$ & $\begin{array}{c}20.66 \\
(0.017)^{* * *}\end{array}$ & $\begin{array}{c}9.56 \\
(0.011)^{* *}\end{array}$ \\
\hline PS & & & & $\begin{array}{c}3.39 \\
(\mathbf{0 . 0 7 8})^{* * * *}\end{array}$ & $\begin{array}{c}12.16 \\
(\mathbf{0 . 0 7 8})^{* * *}\end{array}$ & $\begin{array}{c}4.25 \\
(0.121)\end{array}$ \\
\hline Constant & $\begin{array}{c}517.75 \\
(0.000)^{*}\end{array}$ & $\begin{array}{l}2397.94 \\
(0.000)^{*}\end{array}$ & $\begin{array}{c}378.62 \\
(\mathbf{0 . 0 0 0})^{*}\end{array}$ & $\begin{array}{c}-3.41 \\
(\mathbf{0 . 3 8 4})\end{array}$ & & $\begin{array}{l}-2105.97 \\
(0.000)^{*}\end{array}$ \\
\hline Trend & $\begin{array}{c}33.67 \\
(0.000)^{*}\end{array}$ & & $\begin{array}{c}35.51 \\
(\mathbf{0 . 0 0 0})^{*}\end{array}$ & $\begin{array}{c}19.64 \\
(0.334)\end{array}$ & & \\
\hline
\end{tabular}

جدول (6): نتائج تقدير معاملات الأجل القصير (صيغة تصحيح الخطأ)

\begin{tabular}{lccc|ccc}
\hline & \multicolumn{3}{c}{ Model (1) } & \multicolumn{3}{c}{ Model (2) } \\
\cline { 2 - 7 } & CPI & CC & ICRG & CPI & CC & ICRG \\
\hline dCor & -21.06 & -1.78 & 13.46 & -23.95 & -0.771 & 15.65 \\
& $(\mathbf{0 . 0 8 4})^{* * * *}$ & $(\mathbf{0 . 0 2 6})^{* *}$ & $(\mathbf{0 . 0 3 6})^{* *}$ & $(\mathbf{0 . 0 0 2})^{*}$ & $(\mathbf{0 . 0 0 9})^{*}$ & $(\mathbf{0 . 0 0 0})^{*}$ \\
dCor(-1) & & 2.34 & & & 1.27 & -17.49 \\
& & $(0.000)^{*}$ & & & $(0.000)^{*}$ & $(\mathbf{0 . 0 0 0}) *$
\end{tabular}




\begin{tabular}{|c|c|c|c|c|c|c|}
\hline dGFCF & & & & $\begin{array}{c}0.952 \\
(0.000)^{*}\end{array}$ & $\begin{array}{c}0.408 \\
(0.003)^{*}\end{array}$ & $\begin{array}{c}0.247 \\
(0.046)^{* * *}\end{array}$ \\
\hline dHDI & & & & $\begin{array}{c}600.03 \\
(0.060)^{* * * *}\end{array}$ & $\begin{array}{c}675.08 \\
(0.000)^{*}\end{array}$ & $\begin{array}{c}898.61 \\
(0.000)^{*}\end{array}$ \\
\hline dTRD & & & & $\begin{array}{c}-0.809 \\
(0.132)\end{array}$ & $\begin{array}{c}1.40 \\
(0.002)^{*}\end{array}$ & $\begin{array}{c}2.31 \\
(0.000)^{*}\end{array}$ \\
\hline dPS & & & & $\begin{array}{c}0.278 \\
(0.343) \\
\end{array}$ & $\begin{array}{c}0.227 \\
(0.129) \\
\end{array}$ & $\begin{array}{r}-0.045 \\
(0.777) \\
\end{array}$ \\
\hline ecm (-1) & $\begin{array}{c}-0.384 \\
(0.000)^{*}\end{array}$ & $\begin{array}{c}-0.159 \\
(0.000)^{*}\end{array}$ & $\begin{array}{c}-0.533 \\
(0.000)^{*}\end{array}$ & $\begin{array}{c}-0.345 \\
(0.000)^{*}\end{array}$ & $\begin{array}{c}-0.076 \\
(0.000)\end{array}$ & $\begin{array}{c}-\mathbf{0 . 2 0 7} \\
(\mathbf{0 . 0 0 0})^{*}\end{array}$ \\
\hline
\end{tabular}

من خلال تحليل نتائج الجدول (5) نجد أن مؤشر مدركات الفساد (CPI)، ومؤشر التحكم في الفساد (CC) جاء ليؤكد كل منهما الأخر، بعكس مؤشر الفساد (ICRG) كما يلي: بالنسبة للنموذج الأول: نجد معنوية مؤشر مدركات الفساد (CPI) في تأثير ها على متوسط نصيب الفرد من الناتج المحلى (GDPc) في الأجل الطويل، حيث إن احتمالية t بلغت 0.005، ونتير إثارة المعلمة إلى أن هذا التأثير موجب، فالزيادة في قيمة مؤشر الفساد (CPI) بمقدار درجة واحدة (أي انخفاض مستوى الفساد وزيادة الثفافية بدرجة واحدة)، ينتج عنها انخفاض في منوسط نصيب الفرد من الناتج بمقدار \$158.8 وهو ما يؤكده مؤشر التحكم في الفساد (CC) حيث جاء تأثثره موجب ومعنوي عند 1\% أيضاً، وفيه نجد الزيادة بمقدار درجة واحدة (أي انخفاض مستوى الفساد وزيادة الثفافية)، تؤدى إلى انخفاض منوسط نصيب الفرد من الناتج بمقدار \$25.13، و عند تحويل المؤشر ليكون تدرجه من (صفر-10) مثل مؤشر مدركات الفساد يكون حجم التأثير \$251.3. و على عكس المؤشرين السابقين جاء مؤشر الفساد الثالث (ICRG) ذو تأثثر سالب ومعنوي عند مستوى 1\%، و هو يفيد بأن الزيادة في مستوى الفساد بدرجة واحدة يؤدى إلى انخفاض الناتج المحلى بمقدار \$26.9، وقد يرجع ذلك إلى اختلاف منهجية قياس هذا المؤشر عن المؤشرين الآخرين. حيث يركز مؤشر (ICRG) أكثر على الفساد الكبير أو السياسي مثل فساد الانتخابات و التمويل السري للأحزاب و العلاقات الوثيقة والمريبة بين السياسة والأعمال و غيرها، بعكس 
المؤشرين الآخرين حيث ينصب اهتمامهم بصفة أساسية على الفساد الصغير كالرشوة و الححسوبية في الأجزة الحكومية وغير ها من المظاهر.

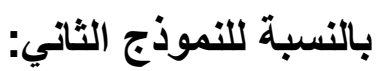
فبعد أخذ التأثير المباشر للفساد والغير مباثر من خلال تأثير الفساد على محددات النمو (قنوات

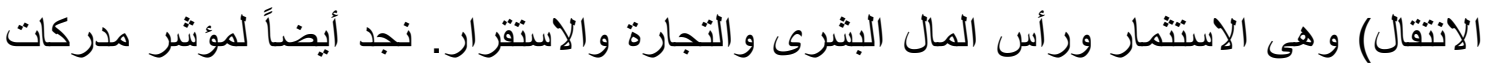

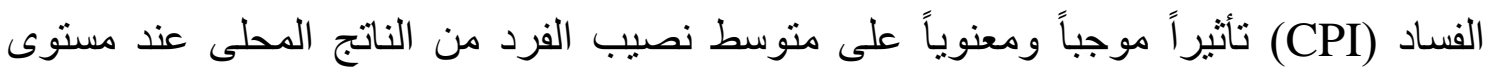
معنوية 5\%، فالزيادة في قيمته بمقدار درجة واحدة (أي انخفاض مستوى الفساد وزيادة مستوى الثفافية)، يؤدى إلى انخفاض متوسط نصيب الفرد من الناتج بمقدار 187.5\$. وهو ما يؤكده مؤشر التحكم في الفساد (CC)، حيث جاء تأثيره موجب ومعنوي أيضاً، وفيه نجد الزيادة بمقدار درجة واحدة (أي انخفاض مستوى الفساد وزيادة الثفافية)، تؤدى إلى انخفاض منوسط نصيب الفرد من الناتج بمقدار 15.9\$، أو 159.1\$ عندما يكون تدرجه من (صفر-10). بينما كان المؤشر الثالث (ICRG) ذو تأثثر سالب ومعنوي على متوسط نصيب الفرد من

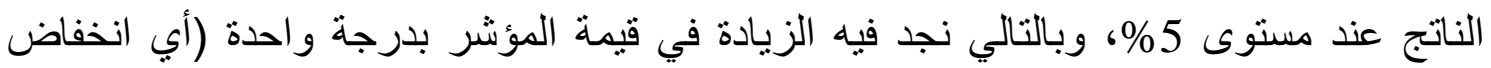

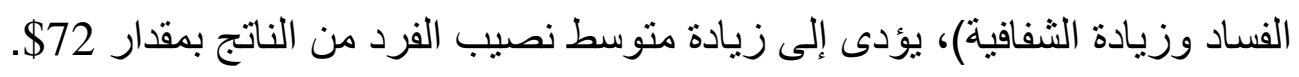
أما بالنسبة لجدول (6) فيتضح منه أن نتائج المعلمات في الأجل القصير تتسق مع نتائجها في الأجل الطويل. كما يعبر معامل تصحيح الخطأ (1-(ecm عن سرعة التكيف من الأجل القصير إلى الأجل الطويل (أي يقيس سرعة تعديل الاختلال في التوازن من الأجل القصير باتجاه التوازن

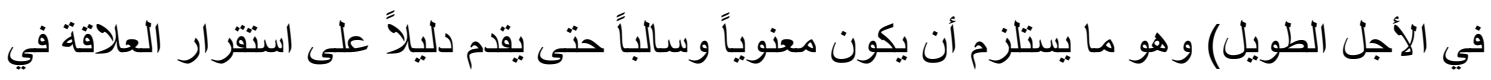

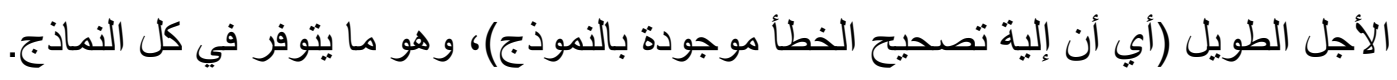

\section{(3-5) اختبار استقرار النماذج (Stability Test):}

لكي نتأكد من خلو البيانات المستخدمة في هذه الدراسة من وجود أي تغير ات هيكلية فيها (أي

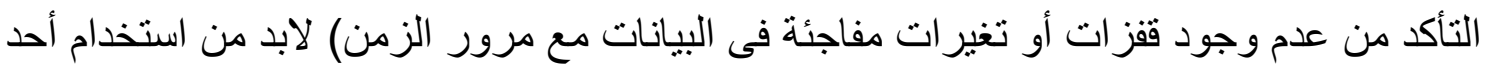

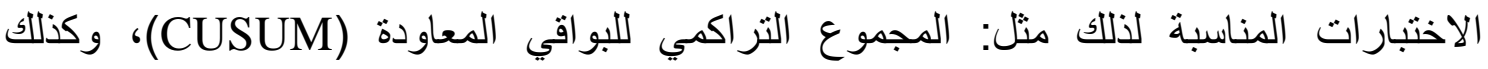

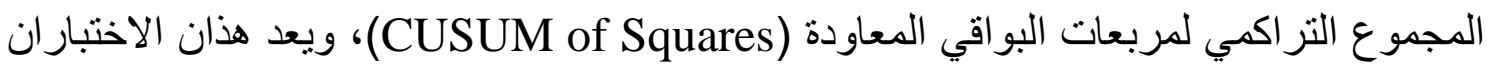
من أهم الاختبارات في هذا المجال لأنهما يوضحا أمرين هامين وهما تبيان وجود أي تغير هيكلي 
فى البيانات، ومدى استقرار وانسجام المعلمات طويلة الأجل مع المعلمات قصبرة الأجل. وقد أظهرت الكثير من الدراسات أن مثل هذه الاختبارات دائماً نجدها مصاحبة لمنهجية ARDL. ويتحقق الاستقرار الهيكلي للمعاملات المقدرة لصيغة تصحيح الخطأ لنموذج (ARDL)، إذا وقع الثكل البياني لاختبار (CUSUM) و (CUSUM of Squares) داخل الحدود الحرجة عند مستوى 5\%. و على ضوء ذللك قمنا بتطبيق اختبار CUSUM كما بالثكل التالي:

شكل (1): اختبار المجموع التراكمي للبواقي المعاودة (CUSUM) لمعادلات الدراسة

Eq4،Model 2

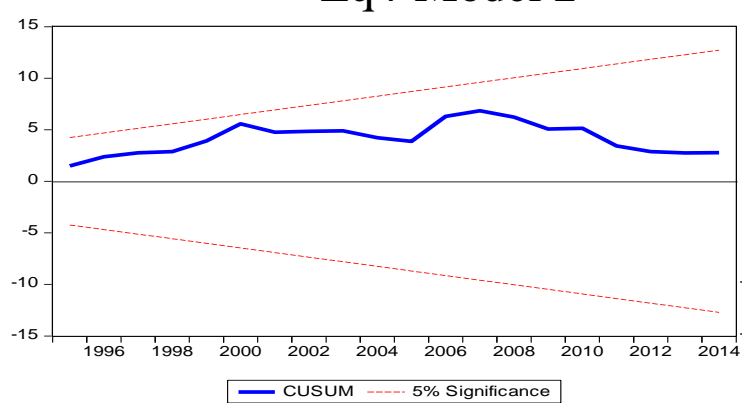

Eq5،Model 2

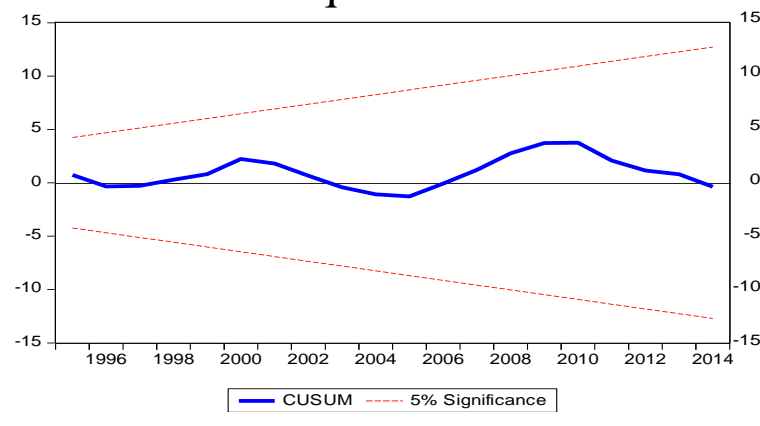

Eq6‘Model 2
Eq1،Model 1

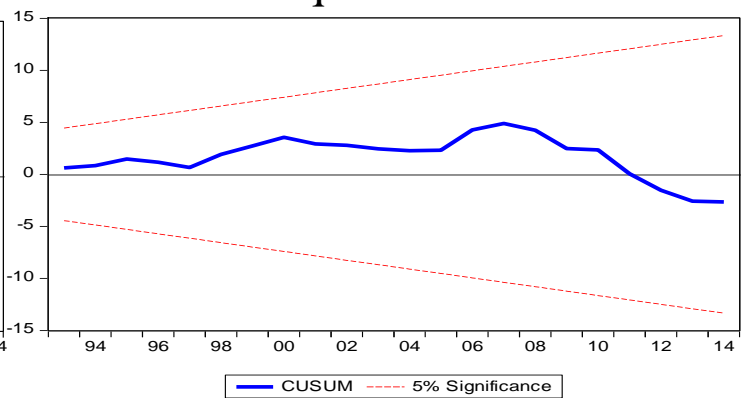

$\mathrm{Eq} 2$

‘Model 1

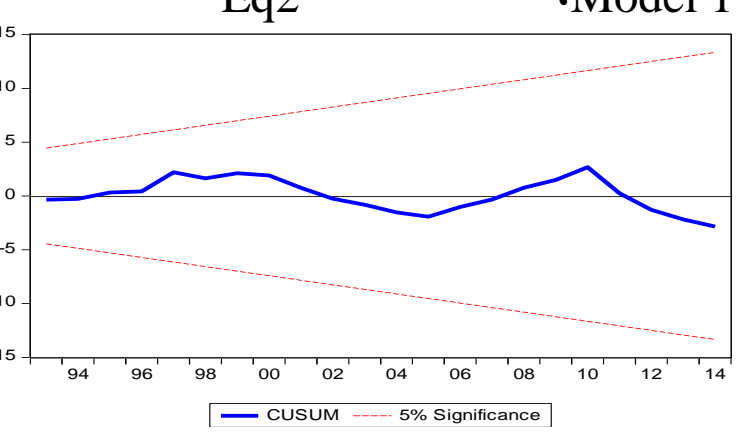

Eq3

‘Model 1 

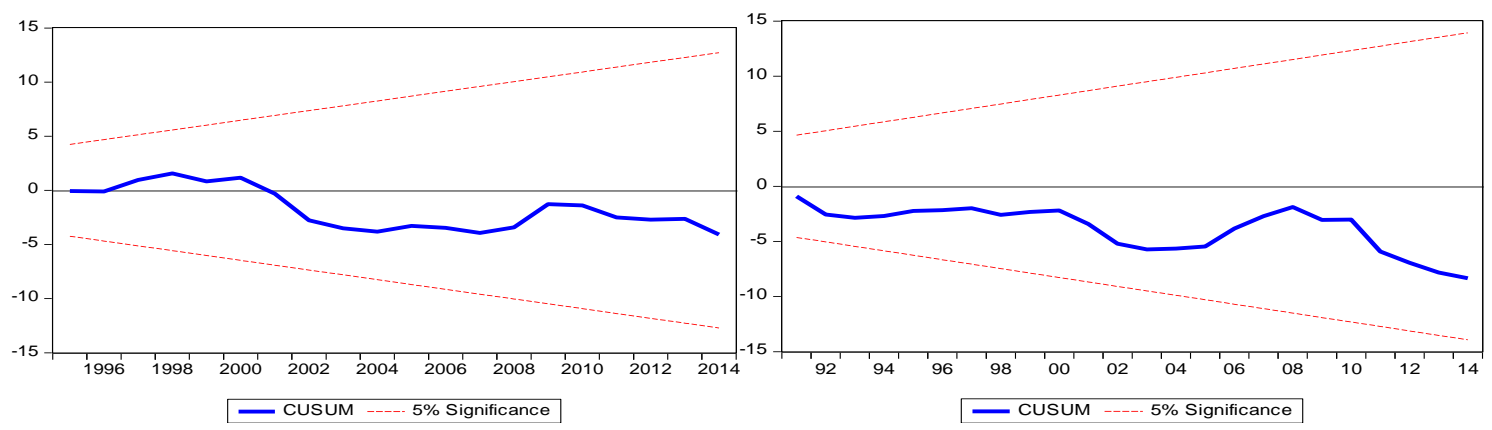

من خلال الرسم البياني نلاحظ أن اختبار (CUSUM) بالنسبة لجميع النماذج يعبر وسط خطى داخل حدود المنطقة الحرجة مشيراً إلى نوع من الاستقرار فى النماذج عند حدود معنوية

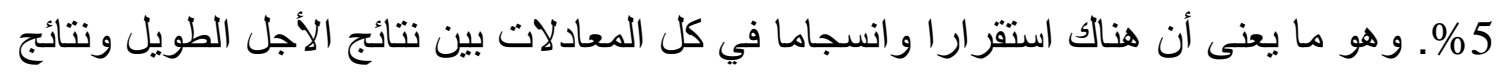

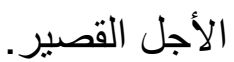

\section{(4-5) (ختبار قوة العلاقة (Robustness Checks)}

نتيجة لتشكيك بعض الباحثين من قوة العلاقة التطبيقية بين الفساد والنمو، فقد قامت الدراسة

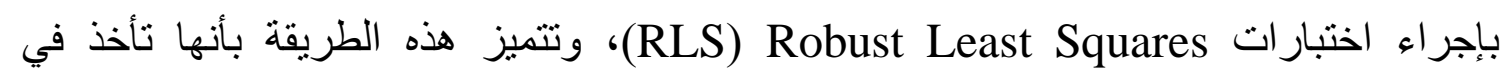
اعتبار ها غياب بعض فروض المربعات الصغرى العادية أو في حالة وجود قيم منطرفة (شاذة). وقد تم تقدير العلاقة بين الفساد والنمو الاقتصادي في مصر باستخدام هذه الطريقة، كما في

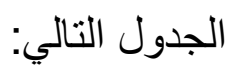

جدول (7): نتائج اختبار علاقة الفساد بالنمو باستخدام (RLS)

\begin{tabular}{|c|c|c|c|c|c|c|}
\hline & \multicolumn{3}{|c|}{ Model (1) } & \multicolumn{3}{|c|}{ Model (2) } \\
\hline & CPI & $\mathrm{CC}$ & ICRG & CPI & $\mathrm{CC}$ & ICRG \\
\hline Corruption & $\begin{array}{c}-86.81 \\
(0.006) *\end{array}$ & $\begin{array}{c}-21.84 \\
(0.000)^{*}\end{array}$ & $\begin{array}{c}10.08 \\
(0.458)\end{array}$ & $\begin{array}{c}-103.89 \\
(0.018)^{* * *}\end{array}$ & $\begin{array}{c}-4.56 \\
(0.011)^{* *}\end{array}$ & $\begin{array}{c}19.49 \\
(0.230)\end{array}$ \\
\hline GFCF & & & & $\begin{array}{c}-1.29 \\
(0.159)\end{array}$ & $\begin{array}{c}-1.21 \\
(0.305)\end{array}$ & $\begin{array}{c}-0.89 \\
(0.380)\end{array}$ \\
\hline HDI & & & & $\begin{array}{l}3597.73 \\
(0.000)^{*}\end{array}$ & $\begin{array}{l}2402.07 \\
(0.000)^{*}\end{array}$ & $\begin{array}{l}3624.99 \\
(0.000)^{*}\end{array}$ \\
\hline TRD & & & & $\begin{array}{c}2.56 \\
(0.079) * * *\end{array}$ & $\begin{array}{c}2.63 \\
(0.134)\end{array}$ & $\begin{array}{c}4.01 \\
(0.006) *\end{array}$ \\
\hline PS & & & & $\begin{array}{c}-1.74 \\
(0.214)\end{array}$ & $\begin{array}{c}-3.52 \\
(0.015)^{* * *}\end{array}$ & $\begin{array}{c}-1.64 \\
(0.286)\end{array}$ \\
\hline
\end{tabular}




$\begin{array}{cccccc}\text { Constant } & 1076.57 & 2125.79 & 774.21 & -632.38 & -1069.33 \\ & (0.000)^{*} & (0.000)^{*} & (0.000)^{*} & (0.019)^{* *} & (0.000) *\end{array}$

\section{Trend}

33.73

(0.000)*

34.08

(0.000)*

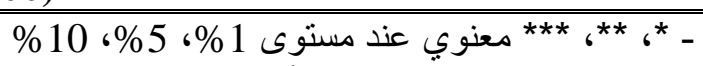

يتضح من نتائج الجدول أتساق تقديرات المعلمات التي تم الحصول عليها باستخدام طريقة

مع تقديرات المعلمات التي تم الحصول عليها باستخدام طريقة (RLS) تأثثر مؤشري الفساد (CPI)، (CC) إيجابي ومعنوي على النمو الاقتصادي، كما جاء تأثير مؤشر الفساد (ICRG) سلبي على النمو ولكنه كان غير معنوي.

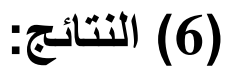

تشير نتائج الدراسة إلى أن الفساد له تأثثير إيجابي على النمو الاقتصادي في مصر، وذلك باستخدام مؤشري مدركات الفساد (CPI)، والتحكم في الفساد (CC)، مما يدعم فرضية (Sand in the Wheels) (Grease the Wheel) إلا من خلال مؤشر الفساد (ICRG)، والذي جاء تأثثره سلبي على النمو الاقتصادي في مصر، وقد يرجع ذلك إلى اختلاف منهجية قياس هذا المؤشر عن المؤشرين الآخرين. حيث يركز مؤشر أكثر على الفساد الكبير أو السياسي مثل فساد الانتخابات والتمويل السري للأحزاب (ICRG) و العلاقات الوثيقة والمريبة بين السياسة والأعمال وغيرها، بعكس المؤشرين الآخرين حيث ينصب اهتمامهم بصفة أساسية على الفساد الصغير كالرشوة والمحسوبية في الأجهزة الحكومية و غير ها من المظاهر.

بينما يرجع التأثير الإيجابي للفساد ، قد يرجع إلى انخفاض الحكم الرشيد في مصر وعدم تمتعها بمؤسسات سياسية أو اقتصادية قوية بالإضافة لضعف سيادة القانون وانخفاض الحرية الاقتصادية، مما يجعل الفساد بمثابة القناة الخلفية التي يتم من خلالها الإسراع بالإجراءات البيروقر اطية المعقدة، كما يؤدى إلى تقليل عنصر المخاطرة و عدم التأكد في العملية الاستثمارية نتيجة لنقص المعلومات و التغيرات الحادة في ظروف الطلب و العرض وضعف المرونة، وكذلك عدم القدرة على التنبؤ بسلوك الحكام تجاههم في المستقبل خاصة في ظل الطابع الثخصي للحكم في مصر. 
عزت، أسماء محمد (2011). إثكالية الفساد والنمو الاقتصادى_دراسة تطبيقية على الدول النامية"، رسالة ماجستير، كلية الاقتصاد، جامعة القاهرة.

Aidt، T. S. (2009). Corruption، institutions، and economic development، Oxford Review of Economic Policy، 25(2)، 271-291.

Ali، N.، \& Comm، B. (2008). Corruption and Its Impact on Economic Growth: Is East Asia Special?، Ph.D Thesis، University of Western Australia.

Bissessar، N. (2008). Corruption: Its Persistence، Causes and Consequences. Ph‘D Theses، Clark University، Worcester.

Dridi، M. (2013). Corruption and economic growth: the transmission channels، Journal of Business Studies Quarterly، 4(4)، 2152-1034.

Dzhumashev، R. (2009). Is there a direct effect of corruption on growth?، MPRA Paper No. 18489، posted 10.

Gyimah-Brempong، K.، \& de Gyimah-Brempong، S. M. (2006). Corruption، growth، and income distribution: Are there regional differences?، Economics of Governance، 7(3)، 245-269.

Hodge، A.، Shankar، S.، Rao، D. S.، \& Duhs‘ A. (2011). Exploring the links between corruption and growth' Review of Development Economics، 15(3)، 474-490.

Lambsdorff‘ J. G. (2002). Corruption and rent-seeking، Public Choice، 113(1-2)، 97-125.

Lash، N. A. (2004). Corruption and Economic Development، The Journal of Economic Asymmetries، 1(1).

Méndez‘ F.، \& Sepúlveda، F. (2006). Corruption‘ growth and political regimes: cross country evidence، European Journal of Political Economy، 22(1)، 82-98. 
Mobolaji، H. I.، \& Omoteso، K. (2009). Corruption and economic growth in some selected transitional economies'Social Responsibility Journal، 5(1)، 70-82.

Nuriyev، G.`\& Hickson، C. (2012). Corruption and Economic Development‘ Available at SSRN 2063065.

Shera' A.6 Dosti، B.، \& Grabova' P. (2014). Corruption impact on Economic Growth: An empirical analysis، Journal of Economic Development · Management · IT ، Finance \& Marketing ، 6(2).

Venard، B. (2013). Institutions، Corruption and Sustainable Development.Economics، Bulletin، 33(4)، 2545-2562.

Wei، S. J.، \& Zeckhauser، R. (1999). Dark deals and dampened destinies: corruption and economic performance 'Japan and the World Economy، 11(3)، 443-454.

Wisitsuwan‘ W.، \& Chintrakarn‘ P. (2012). Human Development، Corruption and Economic Development: Cross-Country Evidence. European Journal of Scientific Research، 74(3)‘469474.

\section{Abstract:}

This paper studies the effect of corruption on long-run growth in Egypt during the period (1990-2014)، based on the methodology Co integration using The Bounds Testing Approach and building on The Autoregressive Distributed Lag (ARDL). Unlike previous empirical studies.

the results have supported both of positive and negative impact of corruption, where the results showed a positive effect of corruption on economic growth using two indicators of corruption they corruption perception index ' and control of corruption. Which supports the hypothesis 
(Grease the Wheel)، while came the impact of corruption index (ICRG) negativer which supports the hypothesis (Sand in the Wheels). These results are robust against different tests (Robustness).

Keywords: corruption، economic growth، ARDL method، RLS method. 